doi:10.17659/01.2019.0057

Journal of Case Reports 2019;9(4):214-216

\title{
Takayasu Arteritis Presenting with Acute Critical Ischemia of Bilateral Lower Limbs
}

\author{
Mohd Al-Baqlish Mohd Firdaus ${ }^{1}$, Mohammad Arif Shahar ${ }^{1}$, Ainon Mohd Mokhtar², Hilmi Abdullah ${ }^{1}$ \\ ${ }^{1}$ Department of Internal Medicine, Kulliyyah of Medicine, International Islamic University Malaysia, Jalan Hospital Campus, \\ 25150 Kuantan Pahang, Malaysia; ${ }^{2}$ Department of Medicine, Hospital Tengku Ampuan Afzan, 25150 Kuantan Pahang, Malaysia.
}

Corresponding Author:

Dr. Mohd Al-Baqlish Bin Mohd Firdaus

Email: baqlish@iium.edu.my

This is an Open Access article distributed under the terms of the Creative Commons Attribution License (creativecommons.org/ licenses/by/3.0).

\begin{abstract}
Background: Takayasu arteritis is a rare systemic vasculitis affecting large arteries. Case Report: In this case report, we will be discussing an uncommon presentation of Takayasu arteritis with critical limbs ischemia of bilateral lower limbs. Conclusion: Takayasu arteritis should be suspected in a young patient presenting with critical limb ischemia especially in a patient without traditional risk factor for atherosclerosis and cardio-embolism. We also share our experience in managing the acute presentation of Takayasu arteritis in our setting.
\end{abstract}

Keywords: Atherosclerosis, Embolism, Ischemia, Systemic Vasculitis, Takayasu Arteritis.

\section{Introduction}

Takayasu arteritis is a rare systemic vasculitis of the large arteries. To the best of our knowledge, this is one of the few reported cases of Takayasu arteritis presenting with bilateral lower limb critical limb ischemia.

\section{Case Report}

Madam A, 37 years old Malay lady with no significant past medical history was referred from a district hospital for progressive and worsening pain associated with bluish and dusky discoloration of the left foot for three days. The pain was sudden in onset, pricking in nature and associated with numbness; and reduced sensation in the affected area. She was unable to walk for last one day. The upper limb was normal. She also complained of low-grade fever for the past one month. There was no history of Raynaud's phenomena or any other symptoms suggestive of connective tissue disease. She has no known drug or food allergies. On further questioning, she had a history of taking hormonal therapy for infertility two years ago for three months however denied any traditional or over the counter medications. On initial examination, her foots were bluish and dusky in color up to the level of malleoli. There was no ulcer or gangrenous patch. The sensation to light touch and pin prick were reduced upto the mid-feet region bilaterally. She had needle pricking like pain upon passive movement of the toes and refused to move them. The capillaries refill time was delayed.

Doppler examination of the lower limbs shows no signal detected over both bilateral dorsalis pedis and posterior tibialis arteries. She was diagnosed with the peripheral arterial disease and scheduled for bilateral below knee amputation. However pre-operatively she was seen by our team and the other peripheral pulses were examined. We also noticed that her blood pressure on the right arms was lower than her left arm (130/60 vs $110 / 50 \mathrm{mmHg}$ ). Her diagnosis was revised to Takayasu arteritis. She was started on intravenous methylprednisolone $500 \mathrm{mg}$ daily. Two days after 
that her peripheral pulses were re-examined. Her foot's pain had disappeared and she was able to walk after a week of treatment. CT-angiogram of the lower limbs after starting of treatment showed narrowing of the bilateral common iliac, external iliac and femoral arteries. The superficial femoral arteries and popliteal arteries are all small [Fig.1].

She is currently still under rheumatology clinic follow up and had no active complaint. Her pulses are all palpable. She was last seen in our rheumatology clinic in May 2017 and currently only on azathioprine $100 \mathrm{mg}$ daily.

\section{Discussion}

Takayasu arteritis is an uncommon vasculitis affecting the large vessel especially the pulmonary arteries; aorta and its main branches [1]. It's common among female compared to male and it usually affects young population [2]. In Chinese population, Takayasu arteritis usually presented with non-specific symptoms i.e. fever, malaise, loss of weight, limb claudication, hypertension and vessel bruits [3]. Our patient presented with critical ischemia of bilateral lower limbs which was a rare presentation of Takayasu arteritis. Misra et al. reported cases of Takayasu arteritis who presented with lower limb gangrene and four of the patients were amputated [4]. Our patient was lucky as a timely diagnosis and treatment were able to save her limbs from amputation. The American College of Rheumatology proposed a set of criteria in 1990 for clinical diagnosis of Takayasu arteritis, which requires three out of six criteria to diagnose TA [5].

Takayasu arteritis is a clinical diagnosis and needs a high level of suspicion in establishing the diagnosis [6]. Our patient fulfilled the criteria of American College of Rheumatology 1990. The only radiology imaging that we had for her was the CTangiogram of bilateral lower limbs as mentioned above. CT-angiogram showed narrowing of the bilateral common iliac arteries which are branches of the aorta. Further attempt to counsel for imaging

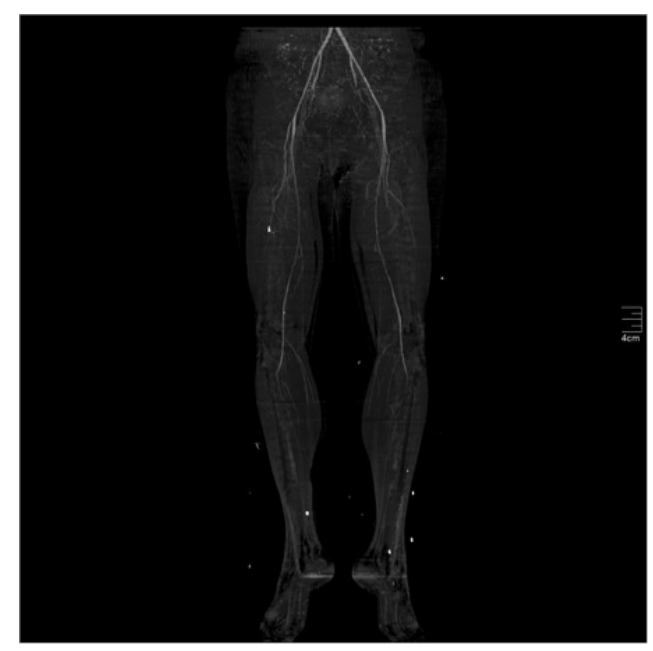

Fig.1: CT-angiogram of the bilateral lower limbs.

of the aorta and its branches was refused by the patient.

The mainstay treatments for Takayasu arteritis are corticosteroid and immunosuppression. The dosage of corticosteroid used in Takayasu arteritis is $1 \mathrm{mg} / \mathrm{kg} /$ day of prednisolone or equivalent [6]. The dosage of prednisolone subsequently has to be tapered down and most physicians will overlap conventional immunosuppressive agents e.g. methotrexate, azathioprine, cyclophosphamide and mycophenolate mefotil while the patient on a tapering dose of prednisolone [6,7]. Our patient was started on oral prednisolone and azathioprine and had tremendous response to treatment and to date there was no sign or symptoms of relapse.

Refractory disease was defined by persistent angiographic and clinical symptoms after six months despite on optimal dosage of prednisolone and immunosuppressive agent; more than three attacks in a year and patient require surgical intervention due to the progression of the disease [6]. There was multiple evidence of successful usage of biologic agents (infliximab, tocilizumab, and rituximab) in the management of refractory Takayasu arteritis [8-10]. This case illustrates the importance of thorough physical examination. Examinations of pulses in the upper limb had saved 
the patient from bilateral lower limb below knee amputation.

\section{Conclusion}

Takayasu arteritis should be suspected in young patients presenting with bilateral limb ischemia especially when traditional risk factors for atherosclerosis and cardio-embolism are absent.

Contributors: MAMF: manuscript writing, and patient management; MAS: reviewing the literature and patient management; AMM: critical inputs into the manuscript and patient management; HA: reviewing the literature and patient management. MAMF will act as a study guarantor. All authors approved the final version of this manuscript. Funding: None; Competing interests: None stated.

\section{References}

1. Dong, H, Che W, Jiang X, Peng M, Zou Y, Zhang H, $\mathrm{Xu} \mathrm{B}$, et al. An unrecognised presentation of Takayasu arteritis: Superficial femoral artery involvement. Clin. Exp. Rheumatol. 2017;35:83-87.

2. Kerr GS. Takayasu arteritis. Ann. Intern. Med. 1994;120(11):919-929.

3. Li J, Sun F, Chen Z, Yang Y, Zhao J, Li M, et al. The clinical characteristics of Chinese Takayasu's arteritis patients: a retrospective study of 411 patients over 24 years. Arthritis Res. Ther. 2017;19:107.
4. Misra DP, Chowdhury AC, Lal H, Mohindra N, Agarwal V. Gangrene in Takayasu's arteritis: a report of two cases and review of literature. Rheumatol. Int. 2016;36: 449-453.

5. Arend WP, Michel BA, Bloch DA, Hunder GG, Calabrese LH, Edworthy SM, et al. The American College of Rheumatology 1990 criteria for the classification of Takayasu arteritis. Arthritis Rheum. 2010;33:1129-1134.

6. Keser G, Direskeneli H, Aksu K. Management of Takayasu arteritis: A systematic review. Rheumatol (United Kingdom). 2014;53:793-801.

7. Barra L, Liang P, Benseler SM, Cabral DA, Fifi-Mah A, Li Y, et al. Variations in the clinical practice of physicians managing Takayasu arteritis: A nationwide survey. Open Access Rheumatol. Res. Rev. 2017;9:91-99.

8. Nishimoto N, Nakahara H, Yoshio-Hoshino N, Mima T. Successful treatment of a patient with Takayasu arteritis using a humanized anti-interleukin-6 receptor antibody. Arthritis Rheum. 2008;58:1197-1200.

9. Tanaka F, Kawakami A, Iwanaga N, Tamai M, Izumi $\mathrm{Y}$, Aratake $\mathrm{K}$, et al. Infliximab is effective for Takayasu arteritis refractory to glucocorticoid and methotrexate. Intern. Med. 2006;45:313-316.

10. Pazzola G, Muratore F, Pipitone N, Crescentini F, Cacoub P, Boiardi L, et al. Rituximab therapy for Takayasu arteritis: a seven patients experience and a review of the literature. Rheumatology. 2018;57:1311. 